\title{
Orthogonally additive-additive and orthogonally quadratic-quadratic functional equation in orthogonality spaces
}

\author{
Choonkil Park', Gwang Hui Kim², Jung Rye Lee ${ }^{3}$ and Dong Yun Shin ${ }^{4^{*}}$
}

\footnotetext{
* Correspondence: dyshin@uos.ac. $\mathrm{kr}$

${ }^{4}$ Department of Mathematics, University of Seoul, Seoul 130-743, Korea

Full list of author information is available at the end of the article
}

\section{Abstract}

Using fixed point method, we prove the Hyers-Ulam stability of the orthogonally additive-additive and orthogonally quadratic-quadratic functional equation

$$
\begin{gathered}
f\left(\frac{x+y+z}{2}\right)+f\left(\frac{x+y-z}{2}\right)+f\left(\frac{x-y+z}{2}\right)+f\left(\frac{y+z-x}{2}\right) \\
=f(x)+f(y)+f(z)
\end{gathered}
$$

for all $x, y, z$ with $x \perp y$, in orthogonality Banach spaces and in non-Archimedean orthogonality Banach spaces.

Mathematics Subject Classification (2010): Primary 39B55, 46S10, 39B72, 39B52, 54E40, 47H10, 47S10, 26E30, 12J25, 46H25.

Keywords: Hyers-Ulam stability, orthogonally additive-additive and orthogonally quadratic-quadratic functional equation, fixed point, non-Archimedean normed space, orthogonality space

\section{Introduction and preliminaries}

In 1897, Hensel [1] introduced a normed space which does not have the Archimedean property. It turned out that non-Archimedean spaces have many nice applications (see $[2-5])$.

A valuation is a function $|\cdot|$ from a field $K$ into $[0, \infty)$ such that 0 is the unique element having the 0 valuation, $|r s|=|r| \cdot|s|$ and the triangle inequality holds, i.e.,

$$
|r+s| \leq|r|+|s|, \quad \forall r, s \in K \text {. }
$$

A field $K$ is called a valued field if $K$ carries a valuation. Throughout this paper, we assume that the base field is a valued field, hence call it simply a field. The usual absolute values of $\mathbb{R}$ and $\mathbb{C}$ are examples of valuations.

Let us consider a valuation which satisfies a stronger condition than the triangle inequality. If the triangle inequality is replaced by

$$
|r+s| \leq \max \{|r|,|s|\}, \quad \forall r, s \in K,
$$

then the function $|\cdot|$ is called a non-Archimedean valuation, and the field is called a non-Archimedean field. Clearly $|1|=|-1|=1$ and $|n| \leq 1$ for all $n \in \mathbb{N}$. A trivial

(c) 2012 Park et al; licensee Springer. This is an Open Access article distributed under the terms of the Creative Commons Attribution License (http://creativecommons.org/licenses/by/2.0), which permits unrestricted use, distribution, and reproduction in any medium, provided the original work is properly cited. 
example of a non-Archimedean valuation is the function $|\cdot|$ taking everything except for 0 into 1 and $|0|=0$.

Definition 1.1. Let $X$ be a vector space over a field $K$ with a non-Archimedean valuation $|\cdot|$. A function $\|\cdot\|: X \rightarrow[0, \infty)$ is said to be a non-Archimedean norm if it satisfies the following conditions:

(i) $\|x\|=0$ if and only if $x=0$;

(ii) $\| r x||=|r||| x||(r \in K, x \in X)$;

(iii) the strong triangle inequality

$$
\|x+y\| \leq \max \{\|x\|,\|y\|\}, \quad \forall x, y \in X
$$

holds. Then $(X,\|\cdot\|)$ is called a non-Archimedean normed space.

\section{Definition 1.2.}

(i) Let $\left\{x_{n}\right\}$ be a sequence in a non-Archimedean normed space $X$. Then the sequence $\left\{x_{n}\right\}$ is called Cauchy if for a given $\varepsilon>0$ there is a positive integer $N$ such that

$$
\left\|x_{n}-x_{m}\right\| \leq \varepsilon
$$

for all $n, m \geq N$.

(ii) Let $\left\{x_{n}\right\}$ be a sequence in a non-Archimedean normed space $X$. Then the sequence $\left\{x_{n}\right\}$ is called convergent if for a given $\varepsilon>0$ there are a positive integer $N$ and an $x \in X$ such that

$$
\left\|x_{n}-x\right\| \leq \varepsilon
$$

for all $n \geq N$. Then we call $x \in X$ a limit of the sequence $\left\{x_{n}\right\}$, and denote by $\lim _{n \rightarrow \infty} x_{n}=x$.

(iii) If every Cauchy sequence in $X$ converges, then the non-Archimedean normed space $X$ is called a non-Archimedean Banach space.

Assume that $\mathrm{X}$ is a real inner product space and $f: X \rightarrow \mathbb{R}$ is a solution of the orthogonal Cauchy functional equation $f(x+y)=f(x)+f(y),\langle x, y\rangle=0$. By the Pythagorean theorem $f(x)=\|x\|^{2}$ is a solution of the conditional equation. Of course, this function does not satisfy the additivity equation everywhere. Thus orthogonal Cauchy equation is not equivalent to the classic Cauchy equation on the whole inner product space.

Pinsker [6] characterized orthogonally additive functionals on an inner product space when the orthogonality is the ordinary one in such spaces. Sundaresan [7] generalized this result to arbitrary Banach spaces equipped with the Birkhoff-James orthogonality. 
The orthogonal Cauchy functional equation

$$
f(x+y)=f(x)+f(y), \quad x \perp y,
$$

in which $\perp$ is an abstract orthogonality relation, was first investigated by Gudder and Strawther [8]. They defined $\perp$ by a system consisting of five axioms and described the general semi-continuous real-valued solution of conditional Cauchy functional equation. In 1985, Rätz [9] introduced a new definition of orthogonality by using more restrictive axioms than of Gudder and Strawther. Moreover, he investigated the structure of orthogonally additive mappings. Rätz and Szabó [10] investigated the problem in a rather more general framework.

Let us recall the orthogonality in the sense of Rätz; cf. [9].

Suppose $X$ is a real vector space with $\operatorname{dim} X \geq 2$ and $\perp$ is a binary relation on $X$ with the following properties:

(O1) totality of $\perp$ for zero: $x \perp 0,0 \perp x$ for all $x \in X$;

$\left(\mathrm{O}_{2}\right)$ independence: if $x, y \in X-\{0\}, x \perp y$, then $x, y$ are linearly independent;

$\left(O_{3}\right)$ homogeneity: if $x, y \in X, x \perp y$, then $\alpha x \perp \beta y$ for all $\alpha, \beta \in \mathbb{R}$;

$\left(O_{4}\right)$ the Thalesian property: if $P$ is a 2-dimensional subspace of $X, x \in P$ and $\lambda \in \mathbb{R}_{+}$, which is the set of nonnegative real numbers, then there exists $y_{0} \in P$ such that $x \perp y_{0}$ and $x+y_{0} \perp \lambda x-y_{0}$.

The pair $(X, \perp)$ is called an orthogonality space. By an orthogonality normed space we mean an orthogonality space having a normed structure.

Some interesting examples are

(i) The trivial orthogonality on a vector space $X$ defined by $\left(O_{1}\right)$, and for non-zero elements $x, y \in X, x \perp y$ if and only if $x, y$ are linearly independent.

(ii) The ordinary orthogonality on an inner product space $(X,\langle. .\rangle$,$) given by x \perp y$ if and only if $\langle x, y\rangle=0$.

(iii) The Birkhoff-James orthogonality on a normed space $(X,\|\|$.$) defined by x \perp y$ if and only if $\|x+\lambda y\| \geq\|x\|$ for all $\lambda \in \mathbb{R}$.

The relation $\perp$ is called symmetric if $x \perp y$ implies that $y \perp x$ for all $x, y \in X$. Clearly examples (i) and (ii) are symmetric but example (iii) is not. It is remarkable to note, however, that a real normed space of dimension $i^{2}$ is an inner product space if and only if the Birkhoff-James orthogonality is symmetric. There are several orthogonality notions on a real normed space such as Birkhoff-James, Boussouis, Singer, Carlsson, unitary-Boussouis, Roberts, Phythagorean, isosceles and Diminnie (see [11-18]).

The stability problem of functional equations originated from the following question of Ulam [19]: Under what condition does there exist an additive mapping near an approximately additive mapping? In 1941, Hyers [20] gave a partial affirmative answer to the question of Ulam in the context of Banach spaces. In 1978, Rassias [21] extended the theorem of Hyers by considering the unbounded Cauchy difference $\mid f(x$ $+y)-f(x)-f(y) \| \leq \varepsilon\left(\|x\|^{p}+\|y\|^{p}\right),(\varepsilon>0, p \in[0,1))$. During the last decades several stability problems of functional equations have been investigated in the spirit of HyersUlam-Rassias. The reader is referred to [22-25] and references therein for detailed information on stability of functional equations. 
Ger and Sikorska [26] investigated the orthogonal stability of the Cauchy functional equation $f(x+y)=f(x)+f(y)$, namely, they showed that if $f$ is a mapping from an orthogonality space $X$ into a real Banach space $Y$ and $\|f(x+y)-f(x)-f(y)\| \leq \varepsilon$ for all $x, y \in X$ with $x \perp y$ and some $\varepsilon>0$, then there exists exactly one orthogonally additive mapping $g: X \rightarrow Y$ such that $\|f(x)-g(x)\| \leq \frac{16}{3} \varepsilon$ for all $x \in X$.

The first author treating the stability of the quadratic equation was Skof [27] by proving that if $f$ is a mapping from a normed space $X$ into a Banach space $Y$ satisfying $\|f(x+y)+f(x-y)-2 f(x)-2 f(y)\| \leq \varepsilon$ for some $\varepsilon>0$, then there is a unique quadratic mapping $g: X \rightarrow Y$ such that $\|f(x)-g(x)\| \leq \frac{\varepsilon}{2}$. Cholewa [28] extended the Skof's theorem by replacing $X$ by an abelian group $G$. The Skof's result was later generalized by Czerwik [29] in the spirit of Hyers-Ulam-Rassias. The stability problem of functional equations has been extensively investigated by some mathematicians (see [30-46]).

The orthogonally quadratic equation

$$
f(x+y)+f(x-y)=2 f(x)+2 f(y), \quad x \perp y
$$

was first investigated by Vajzovic [47] when $X$ is a Hilbert space, $Y$ is the scalar field, $f$ is continuous and $\perp$ means the Hilbert space orthogonality. Later, Drljević [48], Fochi [49] and Szabó [50] generalized this result. See also [51].

Let $X$ be a set. A function $d: X \times X \rightarrow[0, \infty]$ is called a generalized metric on $X$ if $d$ satisfies

(1) $d(x, y)=0$ if and only if $x=y$;

(2) $d(x, y)=d(y, x)$ for all $x, y \in X$;

(3) $d(x, z) \leq d(x, y)+d(y, z)$ for all $x, y, z \in X$.

We recall a fundamental result in fixed point theory.

Theorem 1.3. [52,53] Let $(X, d)$ be a complete generalized metric space and let $\mathrm{J}: X \rightarrow$ $X$ be a strictly contractive mapping with Lipschitz constant $\alpha<1$. Then for each given element $x \in X$, either

$$
d\left(J^{n} x, J^{n+1} x\right)=\infty
$$

for all nonnegative integers $n$ or there exists a positive integer $n_{0}$ such that

(1) $d\left(J^{n} x, J^{n+1} x\right)<\infty$ for all $n \geq n_{0}$;

(2) the sequence $\left\{J^{n} x\right\}$ converges to a fixed point $y^{*}$ of $J$;

(3) $y^{*}$ is the unique fixed point of $J$ in the set $Y=\left\{y \in X \mid d\left(J^{n_{0}} x, y\right)<\infty\right\}$;

(4) $d\left(y, y^{*}\right) \leq \frac{1}{1-\alpha} d(y$, Jy)for all $y \in Y$.

In 1996, Isac and Rassias [54] were the first to provide applications of stability theory of functional equations for the proof of new fixed point theorems with applications. By using fixed point methods, the stability problems of several functional equations have been extensively investigated by a number of authors (see [55-63]).

This paper is organized as follows: In Sect. 2, we prove the Hyers-Ulam stability of the orthogonally additive-additive and orthogonally quadratic-quadratic functional 
equation

$$
\begin{gathered}
f\left(\frac{x+y+z}{2}\right)+f\left(\frac{x+y-z}{2}\right)+f\left(\frac{x-y+z}{2}\right)+f\left(\frac{y+z-x}{2}\right) \\
=f(x)+f(y)+f(z)
\end{gathered}
$$

in orthogonality spaces for an odd mapping. In Sect. 3, we prove the Hyers-Ulam stability of the orthogonally additive-additive and orthogonally quadratic-quadratic functional equation (1.1) in orthogonality spaces for an even mapping. In Sect. 4, we prove the Hyers-Ulam stability of the orthogonally additive-additive and orthogonally quadratic-quadratic functional equation (1.1) in non-Archimedean orthogonality spaces for an odd mapping. In Sect. 5, we prove the Hyers-Ulam stability of the orthogonally additive-additive and orthogonally quadratic-quadratic functional equation (1.1) in non-Archimedean orthogonality spaces for an even mapping.

\section{Stability of the orthogonally additive-additive and orthogonally quadratic- quadratic functional equation in orthogonality spaces: an odd mapping case}

Throughout this section, assume that $(X, \perp)$ is an orthogonality space and that $(Y,\|\|$.

$Y$ ) is a real Banach space.

In this section, applying some ideas from [23,26], we deal with the stability problem for the orthogonally additive-additive and orthogonally quadratic-quadratic functional equation

$$
\begin{gathered}
D f(x, y, x)=f\left(\frac{x+y+z}{2}\right)+f\left(\frac{x+y-z}{2}\right)+f\left(\frac{x-y+z}{2}\right)+f\left(\frac{y+z-x}{2}\right) \\
-f(x)-f(y)-f(z)=0
\end{gathered}
$$

for all $x, y, z \in X$ with $x \perp y$ in orthogonality spaces: an odd mapping case.

If $f$ is an odd mapping with $D f(x, y, z)=0$, then

$$
2 f\left(\frac{x+y}{2}\right)=f(x)+f(y)
$$

for all $x, y \in X$ with $x \perp y$ and

$$
2 f\left(\frac{x+z}{2}\right)=f(x)+f(z)
$$

for all $x, z \in X$. That is, $f$ is additive and orthogonally additive.

Definition 2.1. An odd mapping $f: X \rightarrow Y$ is called an orthogonally additive-additive mapping if

$$
f\left(\frac{x+y+z}{2}\right)+f\left(\frac{x+y-z}{2}\right)+f\left(\frac{x-y+z}{2}\right)+f\left(\frac{y+z-x}{2}\right)=f(x)+f(y)+f(z)
$$

for all $x, y, z \in X$ with $x \perp y$.

Theorem 2.2. Let $\phi: X^{3} \rightarrow[0, \infty)$ be a function such that there exists an $\alpha<1$ with

$$
\varphi(x, y, z) \leq 2 \alpha \varphi\left(\frac{x}{2}, \frac{y}{2}, \frac{z}{2}\right)
$$

for all $x, y, z \in X$ with $x \perp y$. Let $f: X \rightarrow Y$ be an odd mapping satisfying

$$
\|D f(x, y, z)\|_{Y} \leq \varphi(x, y, z)
$$


for all $x, y, z \in X$ with $x \perp y$. Then there exists a unique orthogonally additive-additive mapping $L: X \rightarrow Y$ such that

$$
\|f(x)-L(x)\|_{Y} \leq \frac{\alpha}{1-\alpha} \varphi(x, 0,0)
$$

for all $x \in X$.

Proof. Putting $y=z=0$ in (2.2), we get

$$
\left\|2 f\left(\frac{x}{2}\right)-f(x)\right\|_{Y} \leq \varphi(x, 0,0)
$$

for all $x \in X$, since $x \perp 0$. So

$$
\left\|f(x)-\frac{1}{2} f(2 x)\right\|_{Y} \leq \frac{1}{2} \varphi(2 x, 0,0) \leq \alpha \varphi(x, 0,0)
$$

for all $x \in X$.

Consider the set

$$
S:=\{h: X \rightarrow Y\}
$$

and introduce the generalized metric on $S$ :

$$
d(g, h)=\inf \left\{\mu \in \mathbb{R}_{+}:\|g(x)-h(x)\|_{Y} \leq \mu \varphi(x, 0,0), \quad \forall x \in X\right\},
$$

where, as usual, $\inf \varphi=+\infty$. It is easy to show that $(S, d)$ is complete (see [64]). Now we consider the linear mapping $J: S \rightarrow S$ such that

$$
J g(x):=\frac{1}{2} g(2 x)
$$

for all $x \in X$.

Let $g, h \in S$ be given such that $d(g, h)=\varepsilon$. Then

$$
\|g(x)-h(x)\|_{Y} \leq \varphi(x, 0,0)
$$

for all $x \in X$. Hence

$$
\|J g(x)-J h(x)\|_{Y}=\left\|\frac{1}{2} g(2 x)-\frac{1}{2} h(2 x)\right\|_{Y} \leq \alpha \varphi(x, 0,0)
$$

for all $x \in X$. So $d(g, h)=\varepsilon$ implies that $d(J g, J h) \leq \alpha \varepsilon$. This means that

$$
d(J g, J h) \leq \alpha d(g, h)
$$

for all $g, h \in S$.

It follows from (2.5) that $d(f, I f) \leq \alpha$.

By Theorem 1.3, there exists a mapping $L: X \rightarrow Y$ satisfying the following:

(1) $L$ is a fixed point of $J$, i.e.,

$$
L(2 x)=2 L(x)
$$


for all $x \in X$. The mapping $L$ is a unique fixed point of $J$ in the set

$$
M=\{g \in S: d(h, g)<\infty\} .
$$

This implies that $L$ is a unique mapping satisfying (2.6) such that there exists a $\mu \in$ $(0, \infty)$ satisfying

$$
\|f(x)-L(x)\|_{Y} \leq \mu \varphi(x, 0,0)
$$

for all $x \in X$;

(2) $d\left(J^{n} f, L\right) \rightarrow 0$ as $n \rightarrow \infty$. This implies the equality

$$
\lim _{n \rightarrow \infty} \frac{1}{2^{n}} f\left(2^{n} x\right)=L(x)
$$

for all $x \in X$;

(3) $d(f, L) \leq \frac{1}{1-\alpha} d(f, J f)$, which implies the inequality

$$
d(f, L) \leq \frac{\alpha}{1-\alpha} .
$$

This implies that the inequality (2.3) holds.

It follows from (2.1) and (2.2) that

$$
\begin{aligned}
\|D L(x, y, z)\|_{Y} & =\lim _{n \rightarrow \infty} \frac{1}{2^{n}}\left\|D f\left(2^{n} x, 2^{n} y, 2^{n} z\right)\right\|_{Y} \\
& \leq \lim _{n \rightarrow \infty} \frac{1}{2^{n}} \varphi\left(2^{n} x, 2^{n} y, 2^{n} z\right) \leq \lim _{n \rightarrow \infty} \frac{2^{n} \alpha^{n}}{2^{n}} \varphi(x, y, z)=0
\end{aligned}
$$

for all $x, y, z \in X$ with $x \perp y$. So

$$
L\left(\frac{x+y+z}{2}\right)+L\left(\frac{x+y-z}{2}\right)+L\left(\frac{x-y+z}{2}\right)+L\left(\frac{y+z-x}{2}\right)=L(x)+L(y)+L(z)
$$

for all $x, y, z \in X$ with $x \perp y$. Since $f$ is odd, $L$ is odd. Hence $L: X \rightarrow Y$ is an orthogonally additive-additive mapping. Thus $L: X \rightarrow Y$ is a unique orthogonally additiveadditive mapping satisfying (2.3), as desired.

From now on, in corollaries, assume that $(X, \perp)$ is an orthogonality normed space.

Corollary 2.3. Let $\theta$ be a positive real number and $p$ a real number with $0<p<1$. Let $f: X \rightarrow Y$ be an odd mapping satisfying

$$
\|D f(x, y, z)\|_{Y} \leq \theta\left(\|x\|^{p}+\|y\|^{p}+\|z\|^{p}\right)
$$

for all $x, y, z \in X$ with $x \perp y$. Then there exists a unique orthogonally additive-additive mapping $L: X \rightarrow Y$ such that

$$
\|f(x)-L(x)\|_{Y} \leq \frac{2^{p} \theta}{2-2^{p}}\|x\|^{p}
$$


for all $x \in X$.

Proof. The proof follows from Theorem 2.2 by taking $\phi(x, y, z)=\theta\left(\|x\|^{p}+\|y\|^{p}+\|\right.$ $\left.z \|^{p}\right)$ for all $x, y, z \in X$ with $x \perp y$. Then we can choose $\alpha=2^{p-1}$ and we get the desired result. $\square$

Theorem 2.4. Let $f: X \rightarrow Y$ be an odd mapping satisfying (2.2) for which there exists a function $\phi: X^{3} \rightarrow[0, \infty)$ such that

$$
\varphi(x, y, z) \leq \frac{\alpha}{2} \varphi(2 x, 2 y, 2 z)
$$

for all $x, y, z \in X$ with $x \perp y$. Then there exists a unique orthogonally additive-additive mapping $L: X \rightarrow Y$ such that

$$
\|f(x)-L(x)\|_{Y} \leq \frac{1}{1-\alpha} \varphi(x, 0,0)
$$

for all $x \in X$.

Proof. Let $(S, d)$ be the generalized metric space defined in the proof of Theorem 2.2. Now we consider the linear mapping $J: S \rightarrow S$ such that

$$
J g(x):=2 g\left(\frac{x}{2}\right)
$$

for all $x \in X$.

It follows from (2.4) that $d(f, I f) \leq 1$. So

$$
d(f, L) \leq \frac{\alpha}{1-\alpha} .
$$

Thus we obtain the inequality (2.8).

The rest of the proof is similar to the proof of Theorem 2.2. $\square$

Corollary 2.5. Let $\theta$ be a positive real number and $p$ a real number with $p>1$. Let $f$ : $X \rightarrow Y$ be an odd mapping satisfying (2.7). Then there exists a unique orthogonally additive-additive mapping $L: X \rightarrow Y$ such that

$$
\|f(x)-L(x)\|_{Y} \leq \frac{2^{p} \theta}{2^{p}-2}\|x\|^{p}
$$

for all $x \in X$.

Proof. The proof follows from Theorem 2.4 by taking $\phi(x, y, z)=\theta\left(\|x\|^{p}+\|y\|^{p}+\|\right.$ $\left.z\right|^{p}$ ) for all $x, y, z \in X$ with $x \perp y$. Then we can choose $\alpha=2^{1-p}$ and we get the desired result.

\section{Stability of the orthogonally additive-additive and orthogonally quadratic- quadratic functional equation in orthogonality spaces: an even mapping case}

Throughout this section, assume that $(X, \perp)$ is an orthogonality space and that $(Y,\|\|$. $Y$ ) is a real Banach space.

In this section, applying some ideas from $[23,26]$, we deal with the stability problem for the orthogonally additive-additive and orthogonally quadratic-quadratic functional equation $D f(x, y, z)=0$, given in the previous section, in orthogonality spaces: an even mapping case. 
If $f$ is an even mapping with $D f(x, y, z)=0$, then

$$
2 f\left(\frac{x+y}{2}\right)+2 f\left(\frac{x-y}{2}\right)=f(x)+f(y)
$$

for all $x, y \in X$ with $x \perp y$ and

$$
2 f\left(\frac{x+z}{2}\right)+2 f\left(\frac{x-z}{2}\right)=f(x)+f(z)
$$

for all $x, z \in X$. That is, $f$ is quadratic and orthogonally quadratic.

Definition 3.1. An even mapping $f: X \rightarrow Y$ is called an orthogonally quadratic-quadratic mapping if

$$
f\left(\frac{x+y+z}{2}\right)+f\left(\frac{x+y-z}{2}\right)+f\left(\frac{x-y+z}{2}\right)+f\left(\frac{y+z-x}{2}\right)=f(x)+f(y)+f(z)
$$

for all $x, y, z \in X$ with $x \perp y$.

Theorem 3.2. Let $\phi: X^{3} \rightarrow[0, \infty)$ be a function such that there exists an $\alpha<1$ with

$$
\varphi(x, y, z) \leq 4 \alpha \varphi\left(\frac{x}{2}, \frac{y}{2}, \frac{z}{2}\right)
$$

for all $x, y, z \in X$ with $x \perp y$. Let $f: X \rightarrow Y$ be an even mapping satisfying $f(0)=0$ and (2.2). Then there exists a unique orthogonally quadratic-quadratic mapping $Q: X \rightarrow Y$ such that

$$
\|f(x)-Q(x)\|_{Y} \leq \frac{\alpha}{1-\alpha} \varphi(x, 0,0)
$$

for all $x \in X$.

Proof. Putting $y=z=0$ in (2.2), we get

$$
\left\|4 f\left(\frac{x}{2}\right)-f(x)\right\|_{Y} \leq \varphi(x, 0,0)
$$

for all $x \in X$, since $x \perp 0$. So

$$
\left\|f(x)-\frac{1}{4} f(2 x)\right\|_{Y} \leq \frac{1}{4} \varphi(2 x, 0,0) \leq \alpha \cdot \varphi(x, 0,0)
$$

for all $x \in X$.

By the same reasoning as in the proof of Theorem 2.2, one can obtain an orthogonally quadratic-quadratic mapping $Q: X \rightarrow Y$ defined by

$$
\lim _{n \rightarrow \infty} \frac{1}{4^{n}} f\left(2^{n} x\right)=Q(x)
$$

for all $x \in X$.

Let $(S, d)$ be the generalized metric space defined in the proof of Theorem 2.2.

Now we consider the linear mapping $J: S \rightarrow S$ such that

$$
J g(x):=\frac{1}{4} g(2 x)
$$

for all $x \in X$. 
It follows from (3.3) that $d(f, I f) \leq \alpha$. So

$$
d(f, Q) \leq \frac{\alpha}{1-\alpha}
$$

So we obtain the inequality (3.1). Thus $Q: X \rightarrow Y$ is a unique orthogonally quadraticquadratic mapping satisfying (3.1), as desired.

Corollary 3.3. Let $\theta$ be a positive real number and $p$ a real number with $0<p<2$. Let $f: X \rightarrow Y$ be an even mapping satisfying $f(0)=0$ and (2.7). Then there exists a unique orthogonally quadratic-quadratic mapping $Q: X \rightarrow Y$ such that

$$
\|f(x)-Q(x)\|_{Y} \leq \frac{2^{p} \theta}{4-2^{p}}\|x\|^{p}
$$

for all $x \in X$.

Proof. The proof follows from Theorem 3.2 by taking $\phi(x, y, z)=\theta\left(\|x\|^{p}+\|y\|^{p}+\|\right.$ $z\|\|^{p}$ ) for all $x, y, z \in X$ with $x \perp y$. Then we can choose $\alpha=2^{p-2}$ and we get the desired result. $\square$

Theorem 3.4. Let $f: X \rightarrow Y$ be an even mapping satisfying (2.2) and $f(0)=0$ for which there exists a function $\phi: X^{3} \rightarrow[0, \infty)$ such that

$$
\varphi(x, y, z) \leq \frac{\alpha}{4} \varphi(2 x, 2 y, 2 z)
$$

for all $x, y, z \in X$ with $x \perp y$. Then there exists a unique orthogonally quadratic-quadratic mapping $Q: X \rightarrow Y$ such that

$$
\|f(x)-Q(x)\|_{Y} \leq \frac{\alpha}{1-\alpha} \varphi(x, 0,0)
$$

for all $x \in X$.

Proof. Let $(S, d)$ be the generalized metric space defined in the proof of Theorem 2.2. Now we consider the linear mapping $J: S \rightarrow S$ such that

$$
J g(x):=4 g\left(\frac{x}{2}\right)
$$

for all $x \in X$.

It follows from (3.2) that $d(f, J f) \leq 1$. So we obtain the inequality (3.4).

The rest of the proof is similar to the proofs of Theorems 2.2 and 3.2.

Corollary 3.5. Let $\theta$ be a positive real number and $p$ a real number with $p>2$. Let $f$ : $X \rightarrow Y$ be an even mapping satisfying $f(0)=0$ and (2.7). Then there exists a unique orthogonally quadratic-quadratic mapping $Q: X \rightarrow Y$ such that

$$
\|f(x)-Q(x)\|_{Y} \leq \frac{2^{p} \theta}{2^{p}-4}\|x\|^{p}
$$

for all $x \in X$.

Proof. The proof follows from Theorem 3.4 by taking $\phi(x, y, z)=\theta\left(\|x\|^{p}+\|y\|^{p}+\|\right.$ $z \|^{p}$ ) for all $x, y, z \in X$ with $x \perp y$. Then we can choose $\alpha=2^{2-p}$ and we get the desired result. $\square$

Let $f_{o}(x)=\frac{f(x)-f(-x)}{2}$ and $f_{e}(x)=\frac{f(x)+f(-x)}{2}$. Then $f_{o}$ is an odd mapping and $f_{e}$ is an even mapping such that $f=f_{o}+f_{e}$.

The above corollaries can be summarized as follows: 
Theorem 3.6. Assume that $(X, \perp)$ is an orthogonality normed space. Let $\theta$ be a positive real number and $p$ a real number with $0<p<1$ or $p>2$. Let $f: X \rightarrow Y$ be a mapping satisfying $f(0)=0$ and (2.7). Then there exist an orthogonally additive-additive mapping $L: X \rightarrow Y$ and an orthogonally quadratic-quadratic mapping $Q: X \rightarrow Y$ such that

$$
\|f(x)-L(x)-Q(x)\|_{Y} \leq\left(\frac{2^{p}}{\left|2-2^{p}\right|}+\frac{2^{p}}{\left|4-2^{p}\right|}\right) \theta\|x\|^{p}
$$

for all $x \in X$.

\section{Stability of the orthogonally additive-additive and orthogonally quadratic- quadratic functional equation in non-Archimedean orthogonality spaces: an odd mapping case}

Throughout this section, assume that $(X, \perp)$ is a non-Archimedean orthogonality space and that $\left(Y,\|.\|_{Y}\right)$ is a real non-Archimedean Banach space. Assume that $|2| \neq 1$.

In this section, applying some ideas from $[23,26]$, we deal with the stability problem for the orthogonally additive-additive and orthogonally quadratic-quadratic functional equation $D f(x, y, x)=0$, given in the second section, in non-Archimedean orthogonality spaces: an odd mapping case.

Theorem 4.1. Let $\phi: X^{3} \rightarrow[0, \infty)$ be a function such that there exists an $\alpha<1$ with

$$
\varphi(x, y, z) \leq|2| \alpha \varphi\left(\frac{x}{2}, \frac{y}{2}, \frac{z}{2}\right)
$$

for all $x, y, z \in X$ with $x \perp y$. Let $f: X \rightarrow Y$ be an odd mapping satisfying

$$
\|D f(x, y, z)\|_{Y} \leq \varphi(x, y, z)
$$

for all $x, y, z \in X$ with $x \perp y$. Then there exists a unique orthogonally additive-additive mapping $L: X \rightarrow Y$ such that

$$
\|f(x)-L(x)\|_{Y} \leq \frac{\alpha}{1-\alpha} \varphi(x, 0,0)
$$

for all $x \in X$.

Proof. Putting $y=z=0$ in (4.1), we get

$$
\left\|2 f\left(\frac{x}{2}\right)-f(x)\right\|_{Y} \leq \varphi(x, 0,0)
$$

for all $x \in X$, since $x \perp 0$. So

$$
\left\|f(x)-\frac{1}{2} f(2 x)\right\|_{Y} \leq \frac{1}{|2|} \varphi(2 x, 0,0) \leq \alpha \varphi(x, 0,0)
$$

for all $x \in X$.

Let $(S, d)$ be the generalized metric space defined in the proof of Theorem 2.2.

Now we consider the linear mapping $J: S \rightarrow S$ such that

$$
J g(x):=\frac{1}{2} g(2 x)
$$

for all $x \in X$.

It follows from (4.4) that $d(f, J f) \leq \alpha$. Thus we obtain the inequality (4.2). 
The rest of the proof is similar to the proof of Theorem 2.2.

From now on, in corollaries, assume that $(X, \perp)$ is a non-Archimedean orthogonality normed space.

Corollary 4.2. Let $\theta$ be a positive real number and $p$ a real number with $0<p<1$. Let $f: X \rightarrow Y$ be an odd mapping satisfying (2.7). Then there exists a unique orthogonally additive-additive mapping $L: X \rightarrow Y$ such that

$$
\|f(x)-L(x)\|_{Y} \leq \frac{|2| \theta}{|2|^{p}-|2|}\|x\|^{p}
$$

for all $x \in X$.

Proof. The proof follows from Theorem 4.1 by taking $\phi(x, y, z)=\theta\left(|| x \mid\left\|^{p}+\right\| y\left\|^{p}+\right\|\right.$ $\left.z||^{p}\right)$ for all $x, y, z \in X$ with $x \perp y$. Then we can choose $\alpha=|2|^{1-p}$ and we get the desired result.

Theorem 4.3. Let $f: X \rightarrow Y$ be an odd mapping satisfying (4.1) for which there exists a function $\phi: X^{3} \rightarrow[0, \infty)$ such that

$$
\varphi(x, y, z) \leq \frac{\alpha}{|2|} \varphi(2 x, 2 y, 2 z)
$$

for all $x, y, z \in X$ with $x \perp y$. Then there exists a unique orthogonally additive-additive mapping $L: X \rightarrow Y$ such that

$$
\|f(x)-L(x)\|_{Y} \leq \frac{1}{1-\alpha} \varphi(x, 0,0)
$$

for all $x \in X$.

Proof. Let $(S, d)$ be the generalized metric space defined in the proof of Theorem 2.2. Now we consider the linear mapping $J: S \rightarrow S$ such that

$$
J g(x):=2 g\left(\frac{x}{2}\right)
$$

for all $x \in X$.

It follows from (4.3) that $d(f, J f) \leq 1$. So

$$
d(f, L) \leq \frac{\alpha}{1-\alpha} .
$$

Thus we obtain the inequality (4.5).

The rest of the proof is similar to the proof of Theorem 2.2.

Corollary 4.4. Let $\theta$ be a positive real number and $p$ a real number with $p>1$. Let $f$ : $X \rightarrow Y$ be an odd mapping satisfying (2.7). Then there exists a unique orthogonally additive-additive mapping $L: X \rightarrow Y$ such that

$$
\|f(x)-L(x)\|_{Y} \leq \frac{|2| \theta}{|2|-|2|^{p}}\|x\|^{p}
$$

for all $x \in X$.

Proof. The proof follows from Theorem4.3 by taking $\phi(x, y, z)=\theta\left(\|x\|^{p}+\|y\|^{p}+\|\right.$ $\left.z||^{p}\right)$ for all $x, y, z \in X$ with $x \perp y$. Then we can choose $\alpha=|2|^{p-1}$ and we get the desired result. $\square$ 


\section{Stability of the orthogonally additive-additive and orthogonally quadratic- quadratic functional equation in non-Archimedean orthogonality spaces: an even mapping case}

Throughout this section, assume that $(X, \perp)$ is a non-Archimedean orthogonality space and that $\left(Y,\|.\| \|_{Y}\right)$ is a real non-Archimedean Banach space. Assume that $|2| \neq 1$.

In this section, applying some ideas from $[23,26]$, we deal with the stability problem for the orthogonally additive-additive and orthogonally quadratic-quadratic functional equation $D f(x, y, z)=0$, given in the second section, in non-Archimedean orthogonality spaces: an even mapping case.

Theorem 5.1. Let $\phi: X^{3} \rightarrow[0, \infty)$ be a function such that there exists an $\alpha<1$ with

$$
\varphi(x, y, z) \leq|4| \alpha \varphi\left(\frac{x}{2}, \frac{y}{2}, \frac{z}{2}\right)
$$

for all $x, y, z \in X$ with $x \perp y$. Let $f: X \rightarrow Y$ be an even mapping satisfying $f(0)=0$ and (4.1). Then there exists a unique orthogonally quadratic-quadratic mapping $Q: X \rightarrow Y$ such that

$$
\|f(x)-Q(x)\|_{Y} \leq \frac{\alpha}{1-\alpha} \varphi(x, 0,0)
$$

for all $x \in X$.

Proof. Putting $y=z=0$ in (4.1), we get

$$
\left\|4 f\left(\frac{x}{2}\right)-f(x)\right\|_{Y} \leq \varphi(x, 0,0)
$$

for all $x \in X$, since $x \perp 0$. So

$$
\left\|f(x)-\frac{1}{4} f(2 x)\right\|_{Y} \leq \frac{1}{|4|} \varphi(2 x, 0,0) \leq \alpha \cdot \varphi(x, 0,0)
$$

for all $x \in X$.

By the same reasoning as in the proof of Theorem 2.2, one can obtain an orthogonally quadratic-quadratic mapping $Q: X \rightarrow Y$ defined by

$$
\lim _{n \rightarrow \infty} \frac{1}{4^{n}} f\left(2^{n} x\right)=Q(x)
$$

for all $x \in X$.

Let $(S, d)$ be the generalized metric space defined in the proof of Theorem 2.2.

Now we consider the linear mapping $J: S \rightarrow S$ such that

$$
J g(x):=\frac{1}{4} g(2 x)
$$

for all $x \in X$.

It follows from (5.3) that $d(f, J f) \leq \alpha$. So

$$
d(f, Q) \leq \frac{\alpha}{1-\alpha} .
$$

So we obtain the inequality (5.1). Thus $Q: X \rightarrow Y$ is a unique orthogonally quadraticquadratic mapping satisfying (5.1), as desired. 
Corollary 5.2. Let $\theta$ be a positive real number and $p$ a real number with $0<p<2$. Let $f: X \rightarrow Y$ be an even mapping satisfying $f(0)=0$ and (2.7). Then there exists a unique orthogonally quadratic-quadratic mapping $Q: X \rightarrow Y$ such that

$$
\|f(x)-Q(x)\|_{Y} \leq \frac{|2|^{2} \theta}{|2|^{p}-|2|^{2}}\|x\|^{p}
$$

for all $x \in X$.

Proof. The proof follows from Theorem 5.1 by taking $\phi(x, y, z)=\theta\left(\|x\|^{p}+\|y\|^{p}+\|\right.$ $z||^{p}$ ) for all $x, y, z \in X$ with $x \perp y$. Then we can choose $\alpha=|2|^{2-p}$ and we get the desired result.

Theorem 5.3. Let $f: X \rightarrow Y$ be an even mapping satisfying (4.1) and $f(0)=0$ for which there exists a function $\phi: X^{3} \rightarrow[0, \infty)$ such that

$$
\varphi(x, y, z) \leq \frac{\alpha}{|4|} \varphi(2 x, 2 y, 2 z)
$$

for all $x, y, z \in X$ with $x \perp y$. Then there exists a unique orthogonally quadratic-quadratic mapping $Q: X \rightarrow Y$ such that

$$
\|f(x)-Q(x)\|_{Y} \leq \frac{1}{1-\alpha} \varphi(x, 0,0)
$$

for all $x \in X$.

Proof. Let $(S, d)$ be the generalized metric space defined in the proof of Theorem 2.2. Now we consider the linear mapping $J: S \rightarrow S$ such that

$$
J g(x):=4 g\left(\frac{x}{2}\right)
$$

for all $x \in X$.

It follows from (5.2) that $d(f, J f) \leq 1$. So we obtain the inequality (5.4).

The rest of the proof is similar to the proofs of Theorems 2.2 and 5.1.

Corollary 5.4. Let $\theta$ be a positive real number and $p$ a real number with $p>2$. Let $f$ : $X \rightarrow Y$ be an even mapping satisfying $f(0)=0$ and (2.7). Then there exists a unique orthogonally quadratic-quadratic mapping $Q: X \rightarrow Y$ such that

$$
\|f(x)-Q(x)\|_{Y} \leq \frac{|2|^{2} \theta}{|2|^{2}-|2|^{p}}\|x\|^{p}
$$

for all $x \in X$.

Proof. The proof follows from Theorem 5.3 by taking $\phi(x, y, z)=\theta\left(\|x\|^{p}+\|y\|^{p}+\|\right.$ $z||^{p}$ ) for all $x, y, z \in X$ with $x \perp y$. Then we can choose $\alpha=|2|^{p-2}$ and we get the desired result. $\square$

The above corollaries can be summarized as follows:

Theorem 5.5. Assume that $(X, \perp)$ is a non-Archimedean orthogonality normed space. Let $\theta$ be a positive real number and $p$ a real number with $0<p<1$ (respectively $p>2$ ). Let $f: X \rightarrow Y$ be a mapping satisfying $f(0)=0$ and (2.7). Then there exist an orthogonally additive-additive mapping $L: X \rightarrow Y$ and an orthogonally quadratic-quadratic mapping $Q: X \rightarrow Y$ such that 


$$
\begin{array}{r}
\|f(x)-L(x)-Q(x)\|_{Y} \leq\left(\frac{|2|}{|2|^{p}-|2|}+\frac{|2|^{2}}{|2|^{p}-|2|^{2}}\right) \theta\|x\|^{p} \\
\left(\text { respectively }\|f(x)-L(x)-Q(x)\|_{Y} \leq\left(\frac{|2|}{|2|-|2|^{p}}+\frac{|2|^{2}}{|2|^{2}-|2|^{p}}\right) \theta\|x\|^{p}\right)
\end{array}
$$

for all $x \in X$.

\section{Acknowledgements}

C. Park was supported by Basic Science Research Program through the National Research Foundation of Korea funded by the Ministry of Education, Science and Technology (NRF-2012R1A1A2004299). D. Y. Shin was supported by Basic Science Research Program through the National Research Foundation of Korea funded by the Ministry of Education, Science and Technology (NRF-2010-0021792).

\section{Author details}

${ }^{1}$ Research Institute for Natural Sciences, Hanyang University, Seoul 133-791, Korea ${ }^{2}$ Department of Mathematics, Kangnam University, Yongin, Gyeonggi 446-702, Korea ${ }^{3}$ Department of Mathematics, Daejin University, Kyeonggi 487711, Korea ${ }^{4}$ Department of Mathematics, University of Seoul, Seoul 130-743, Korea

\section{Authors' contributions}

All authors conceived of the study, participated in its design and coordination, drafted the manuscript, participated in the sequence alignment, and read and approved the final manuscript.

\section{Competing interests}

The authors declare that they have no competing interests.

\section{Received: 13 December 2011 Accepted: 15 June 2012 Published: 15 June 2012}

\section{References}

1. Hensel, K: Ubereine news Begrundung der Theorie der algebraischen Zahlen. Jahresber Deutsch Math Verein. 6, 83-88 (1897)

2. Deses, D: On the representation of non-Archimedean objects. Topology Appl. 153, 774-785 (2005). doi:10.1016/j. topol.2005.01.010

3. Katsaras, AK, Beoyiannis, A: Tensor products of non-Archimedean weighted spaces of continuous functions. Georgian Math J. 6, 33-44 (1999). doi:10.1023/A:1022926309318

4. Khrennikov, A: Non-Archimedean analysis: quantum paradoxes, dynamical systems and biological models. In Mathematics and its Applications, vol. 427,Kluwer, Dordrecht (1997)

5. Nyikos, PJ: On some non-Archimedean spaces of Alexandrof and Urysohn. Topol Appl. 91, 1-23 (1999). doi:10.1016/ S0166-8641(97)00239-3

6. Pinsker, AG: Sur une fonctionnelle dans l'espace de Hilbert. C R (Dokl) Acad Sci URSS. 20, 411-414 (1938)

7. Sundaresan, K: Orthogonality and nonlinear functionals on Banach spaces. Proc Am Math Soc. 34, 187-190 (1972). doi:10.1090/S0002-9939-1972-0291835-X

8. Gudder, S, Strawther, D: Orthogonally additive and orthogonally increasing functions on vector spaces. Pac J Math. 58, 427-436 (1975)

9. Rätz, J: On orthogonally additive mappings. Aequ Math. 28, 35-49 (1985). doi:10.1007/BF02189390

10. Rätz, J, Szabó, G: On orthogonally additive mappings IV. Aequ Math. 38, 73-85 (1989). doi:10.1007/BF01839496

11. Alonso, J, Benítez, C: Orthogonality in normed linear spaces: a survey I. Main properties Extr Math. 3, 1-15 (1988)

12. Alonso, J, Benítez, C: Orthogonality in normed linear spaces: a survey II. Relations between main orthogonalities. Extr Math. 4, 121-131 (1989)

13. Birkhoff, G: Orthogonality in linear metric spaces. Duke Math J. 1, 169-172 (1935). doi:10.1215/S0012-7094-35-00115-6

14. Carlsson, SO: Orthogonality in normed linear spaces. Ark Mat. 4, 297-318 (1962). doi:10.1007/BF02591506

15. Diminnie, CR: A new orthogonality relation for normed linear spaces. Math Nachr. 114, 197-203 (1983). doi:10.1002/ mana. 19831140115

16. Cho, Y, Diminnie, CR, Freese, RW, Andalafte, EZ: Isosceles orthogonal triples in linear 2-normed spaces. Math Nachr. 157, 225-234 (1992)

17. James, RC: Orthogonality in normed linear spaces. Duke Math J. 12, 291-302 (1945). doi:10.1215/50012-7094-45-01223-3

18. James, RC: Orthogonality and linear functionals in normed linear spaces. Trans Am Math Soc. 61, 265-292 (1947). doi:10.1090/S0002-9947-1947-0021241-4

19. Ulam, SM: Problems in Modern Mathematics. Wiley, New York (1960)

20. Hyers, DH: On the stability of the linear functional equation. Proc Natl Acad Sci USA. 27, $222-224$ (1941). doi:10.1073/ pnas.27.4.222

21. Rassias, TM: On the stability of the linear mapping in Banach spaces. Proc Am Math Soc. 72, 297-300 (1978). doi:10.1090/50002-9939-1978-0507327-1

22. Czerwik, S: Stability of Functional Equations of Ulam-Hyers-Rassias Type. Hadronic Press, Palm Harbor (2003)

23. Hyers, DH, Isac, G, Rassias, TM: Stability of Functional Equations in Several Variables. Birkhäuser, Basel (1998)

24. Jung, S: Hyers-Ulam-Rassias Stability of Functional Equations in Mathematical Analysis. Hadronic Press, Palm Harbor (2001)

25. Rassias, TM: Functional Equations, Inequalities and Applications. Kluwer, Dordrecht, Boston (2003) 
26. Ger, R, Sikorska, J: Stability of the orthogonal additivity. Bull Pol Acad Sci Math. 43, 143-151 (1995)

27. Skof, F: Proprietà locali e approssimazione di operatori. Rend Sem Mat Fis Milano. 53, 113-129 (1983). doi:10.1007/ BF02924890

28. Cholewa, PW: Remarks on the stability of functional equations. Aequ Math. 27, 76-86 (1984). doi:10.1007/BF02192660

29. Czerwik, S: On the stability of the quadratic mapping in normed spaces. Abh Math Sem Univ Hamburg. 62, 59-64 (1992). doi:10.1007/BF02941618

30. Czerwik, S: Functional Equations and Inequalities in Several Variables. World Scientific Publishing Company, London (2002)

31. Park, C, Park, J: Generalized Hyers-Ulam stability of an Euler-Lagrange type additive mapping. J Differ Equ Appl. 12, 1277-1288 (2006). doi:10.1080/10236190600986925

32. Eshaghi Gordji, M, Savadkouhi, MB: Stability of a mixed type cubic-quartic functional equation in non-Archimedean spaces. Appl Math Lett. 23, 1198-1202 (2010). doi:10.1016/j.aml.2010.05.011

33. Eshaghi Gordji, M, Savadkouhi, MB: Stability of cubic and quartic functional equations in non-Archimedean spaces. Acta Appl Math. 110, 1321-1329 (2010). doi:10.1007/s10440-009-9512-7

34. Gordji, Eshaghi, Khodaei, H, Khodabakhsh, R: General quartic-cubic-quadratic functional equation in non-Archimedean normed spaces. UPB Sci Bull A. 72(3):69-84 (2010)

35. Eshaghi Gordji, M, Khodaei, H, Khodabakhsh, R: On approximate n-ry derivations. Int J Geom Methods Mod Phys. 8, 485-500 (2011). doi:10.1142/S0219887811005245

36. Eshaghi Gordji, M, Ghaemi, MB, Kaboli Gharetapeh, S, Shams, S, Ebadian, A: On the stability of J*-derivations. J Geom Phys. 60, 454-459 (2010). doi:10.1016/.j.geomphys.2009.11.004

37. Eshaghi Gordji, M, Alizadeh, Z: Stability and superstability of ring homomorphisms on non-Archimedean Banach algebras. Abstr Appl Anal 2011 (2011). Article ID 123656

38. Cho, Y, Park, C, Rassias, TM, Saadati, R: Inner product spaces and functional equations. J Comput Anal Appl. 13, 296-304 (2011)

39. Park, C, Cho, Y, Kenary, HA: Orthogonal stability of a generalized quadratic functional equation in non-Archimedean spaces. J Comput Anal Appl. 14, 526-535 (2012)

40. Saadati, $R, C h o, Y$, Vahidi, J: The stability of the quartic functional equation in various spaces. Comput Math Appl. 60 , 1994-2002 (2010). doi:10.1016/j.camwa.2010.07.034

41. Cho, Y, Park, C, Rassias, TM, Saadati, R: Inner product spaces and functional equations. J Comput Anal Appl. 13, 296-304 (2011)

42. Mohammadi, M, Cho, Y, Park, C, Vetro, P, Saadati, R: Random stability of an additive-quadratic-quartic functional equation. J Inequal Appl 2010 (2010). Article ID 754210

43. Baktash, E, Cho, Y, Jalili, M, Saadati, R, Vaezpour, SM: On the stability of cubic mappings and quadratic mappings in random normed spaces. J Inequal Appl 2008 (2008). Article ID 902187

44. Rassias, TM: On the stability of the quadratic functional equation and its applications. Stud Univ Babeş-Bolyai Math. 43, 89-124 (1998)

45. Rassias, TM: The problem of S.M. Ulam for approximately multiplicative mappings. J Math Anal Appl. 246, 352-378 (2000). doi:10.1006/jmaa.2000.6788

46. Rassias, TM: On the stability of functional equations in Banach spaces. J Math Anal Appl. 251, 264-284 (2000) doi:10.1006/imaa.2000.7046

47. Vajzović, F: Über das Funktional $H$ mit der Eigenschaft: $(x, y)=0 \otimes H(x+y)+H(x-y)=2 H(x)+2 H(y)$. Glasnik Mat Ser III. 2(22):73-81 (1967)

48. Drljević, F: On a functional which is quadratic on A-orthogonal vectors. Publ Inst Math (Beograd). 54, 63-71 (1986)

49. Fochi, M: Functional equations in A-orthogonal vectors. Aequ Math. 38, 28-40 (1989). doi:10.1007/BF01839491

50. Szabó, G: Sesquilinear-orthogonally quadratic mappings. Aequ Math. 40, 190-200 (1990). doi:10.1007/BF02112295

51. Paganoni, L, Rätz, J: Conditional function equations and orthogonal additivity. Aequ Math. 50, 135-142 (1995). doi:10.1007/BF01831116

52. Cădariu, L, Radu, V: Fixed points and the stability of Jensen's functional equation. J Inequal Pure Appl Math 4(1) (2003). Article ID 4

53. Diaz, J, Margolis, B: A fixed point theorem of the alternative for contractions on a generalized complete metric space. Bull Am Math Soc. 74, 305-309 (1968). doi:10.1090/S0002-9904-1968-11933-0

54. Isac, G, Rassias, TM: Stability of $\psi$-additive mappings: Appications to nonlinear analysis. Internat J Math Math Sci. 19 219-228 (1996). doi:10.1155/50161171296000324

55. Cădariu, L, Radu, V: On the stability of the Cauchy functional equation: a fixed point approach. Grazer Math Ber. 346, 43-52 (2004)

56. Cădariu, L, Radu, V: Fixed point methods for the generalized stability of functional equations in a single variable. Fixed Point Theory Appl 2008 (2008). Article ID 749392

57. Jung, Y, Chang, I: The stability of a cubic type functional equation with the fixed point alternative. J Math Anal Appl. 306, 752-760 (2005). doi:10.1016/j.jmaa.2004.10.017

58. Park, C: Fixed points and Hyers-Ulam-Rassias stability of Cauchy-Jensen functional equations in Ba-nach algebras. Fixed Point Theory Appl 2007 (2007). Article ID 50175

59. Eshaghi Gordji, M, Najati, A: Approximately J*-homomorphisms: a fixed point approach. J Geom Phys. 60, 809-814 (2010). doi:10.1016/.j.geomphys.2010.01.012

60. Najati, A, Cho, Y: Generalized Hyers-Ulam stability of the pexiderized Cauchy functional equation in non-Archimedean spaces. Fixed Point Theory Appl 2011 (2011). Article ID 309026

61. Cho, Y, Kang, J, Saadati, R: Fixed points and stability of additive functional equations on the Banach algebras. J Comput Anal Appl. 14, 1103-1111 (2012)

62. Park, C: Generalized Hyers-Ulam-Rassias stability of quadratic functional equations: a fixed point approach. Fixed Point Theory Appl 2008 (2008). Article ID 493751

63. Radu, V: The fixed point alternative and the stability of functional equations. Fixed Point Theory. 4, 91-96 (2003) 
64. Miheț, D, Radu, V: On the stability of the additive Cauchy functional equation in random normed spaces. J Math Anal Appl. 343, 567-572 (2008). doi:10.1016/j.jmaa.2008.01.100

doi:10.1186/1029-242X-2012-139

Cite this article as: Park et al:: Orthogonally additive-additive and orthogonally quadratic-quadratic functional equation in orthogonality spaces. Journal of Inequalities and Applications 2012 2012:139.

Submit your manuscript to a SpringerOpen ${ }^{\odot}$ journal and benefit from:

- Convenient online submission

- Rigorous peer review

- Immediate publication on acceptance

- Open access: articles freely available online

- High visibility within the field

- Retaining the copyright to your article

Submit your next manuscript at $\gg$ springeropen.com 